\title{
Necdin and Neurotrophin Receptors: Interactors of Relevance for Neuronal Resistance to Oxidant Stress
}

\author{
CHRISTOPHER A. INGRAHAM, LARISSA WERTALIK, AND NINA F. SCHOR \\ Department of Pediatrics, University of Rochester Medical Center, Rochester, New York 14642
}

\begin{abstract}
Necdin is a protein known to interact with the neurotrophin receptors, neurotrophic tyrosine kinase receptor type 1 (TrkA) and $75 \mathrm{kD}$ low-affinity neurotrophin receptor (p75NTR). TrkA and p75NTR play roles in development and disease of the nervous system and chemoresistance of nervous system tumors. Necdin deletion is associated with Prader-Willi syndrome. The present studies demonstrate that the effects of necdin on the susceptibility of neuroblastoma cells to oxidant stress are dependent on the ratio of p75NTR to TrkA in the cell. In low p75NTR:TrkA ratio cells, necdin down-regulation decreases sensitivity to oxidant stress and expression of and signaling through TrkA. In high p75NTR:TrkA cells, necdin down-regulation is without effect. The effects of necdin deletion on the developing nervous system may depend on the relative expression of p75NTR and TrkA in the cells of particular regions of the nervous system. (Pediatr Res 69: 279-284, 2011)
\end{abstract}

$\mathrm{N}^{2}$ eurotrophins and their receptors play critical roles during development of the nervous system (1-3). They are the initiation point for signaling pathways that underlie major functions and cell fate decisions of developing neurons (4-8). Understanding these signaling pathways will enhance identification of therapeutic targets for disorders of nervous system development.

For example, necdin, a melanoma antigen gene (MAGE) signaling protein, plays a role in terminal neuronal differentiation and is deficient in patients with Prader-Willi syndrome. The necdin protein binds to the neurotrophin receptors, neurotrophic tyrosine kinase receptor type 1 (TrkA) and $75 \mathrm{kD}$ low-affinity neurotrophin receptor (p75NTR). Interestingly, p75NTR, which functions both independently and in a complex with TrkA, does not bind to TrkA in mouse sensory neurons lacking the necdin gene (9), suggesting that necdin may play a key role in establishing the p75NTR-TrkA complex. Whether necdin deficiency itself or the absence of the p75NTR-TrkA complex resulting from necdin deficiency plays a role in Prader-Willi syndrome is unknown.

Received August 3, 2010; accepted October 19, 2010

Correspondence: Nina F. Schor, M.D., Ph.D., Department of Pediatrics, University of Rochester Medical Center, 601 Elmwood Avenue, Box 777, Rochester, NY 14642; e-mail: nina_schor@urmc.rochester.edu

Supported by a National Institute of Health grant, number NS038569, [to N.F.S.] and by the William H. Eilinger Endowment of Golisano Children's Hospital of the University of Rochester Medical Center.
This article demonstrates the dependence of the effects of necdin knockdown on the cellular p75NTR:TrkA ratio. In cells with a high p75NTR:TrkA ratio, necdin knockdown is without effect on sensitivity to oxidant stress. In cells with a low p75NTR:TrkA ratio, necdin knockdown decreases sensitivity to oxidant stress. The effects of necdin deficiency on nervous system development may therefore depend on the p75NTR:TrkA ratio in specific nervous system regions.

\section{METHODS}

Cell culture. Cell lines studied included rat pheochromocytoma (PC12), mouse neuroblastoma (Neuro-2A), and human neuroblastomas (SH-EP1, SH-SY5Y, and SK-N-AS). All cell lines were grown in DMEM/F12 (1/1) media supplemented with $10 \% \mathrm{wt} / \mathrm{vol}$ fetal bovine serum (Cellgro, Manassas, $\mathrm{VA}$; \#35-011-CV) and 1\% wt/vol penicillin-streptomycin (Cellgro, Manassas, VA; \#30-002-CI). This medium was used for all subsequent cell culture experiments. All lines were plated at 10,000 cells/96-well plate or $10 \mathrm{~cm}$ plate and grown in $5 \% \mathrm{CO}_{2}$ at $37^{\circ} \mathrm{C}$.

Real-time PCR. RNA was isolated from pellets containing $3 \times 10^{6}$ cells using the Qiagen RNeasy Mini Kit and Qiagen QIAshredder (Valencia, CA). Genomic DNA was digested with DNase I (Invitrogen, Carlsbad, CA). The reverse transcriptase reaction was performed using the SuperScript III FirstStrand Synthesis System (Invitrogen) with or without (negative control) reverse transcriptase. Primers specific to necdin (forward: gctggtgcagaaggcgcacga, reverse: gctggtacttcaggtaattc) were used to amplify a 455-bp fragment by PCR $\left(T_{\mathrm{m}}=58,35\right.$ cycles $)$.

siRNA treatment. Necdin siRNA [Santa Cruz Biotechnology, Santa Cruz, CA; Ndn siRNA(h) \#sc-37318; Ndn siRNA(m) \#sc-37319], p75NTR siRNA [Integrated DNA Technologies, Coralville, IA; p75 siRNA(h): gcagaacaccgugugc; Qiagen, Valencia, CA; p75(m) \#SI00230230], TrkA siRNA [TrkA(h) \#sc-36726], and control scrambled siRNA (Santa Cruz Biotechnology, Santa Cruz, CA; Control siRNA-A \#sc-37007) were thawed at room temperature, diluted into $50 \mu \mathrm{L}$ medium, and incubated at room temperature for $5 \mathrm{~min}$. Lipofectamine 2000 (Invitrogen, Carlsbad, CA; \#11668-019) was prepared in culture medium per the manufacturer's instructions and allowed to sit for 5 $\mathrm{min}$ at room temperature. The individual siRNA and Lipofectamine solutions were then combined and allowed to incubate at room temperature for $20 \mathrm{~min}$. This mixture was then added to the cells to give a final siRNA concentration of $20 \mu \mathrm{M}$ (necdin, p75NTR, and respective control siRNAs) or $5 \mu \mathrm{M}$ (TrkA sRNA and respective control). The plates were incubated overnight (18-24 h) at $37^{\circ} \mathrm{C}$. Sister wells of cells were then harvested for protein determination or treated with 6-hydroxydopamine (6-OHDA).

Inflicting oxidant stress: 6-OHDA treatment. The 96-well plates of neuroblastoma cells treated with siRNA were subsequently treated with 0-500 $\mu \mathrm{M}$ 6-OHDA (Sigma Chemical Co.-Aldrich, St. Louis, MO;

Abbreviations: Ndn, necdin; 6-OHDA, 6-hydroxydopamine; p75NTR, 75 $\mathrm{kD}$ low-affinity neurotrophin receptor; PC12, a rat pheochromocytoma cell line; SH-EP1, a human neuroblastoma cell line; SH-SY5Y, a human neuroblastoma cell line; SK-N-AS, a human neuroblastoma cell line; TrkA, neurotrophic tyrosine kinase receptor type 1 
\#162957). Briefly, $10 \mathrm{mg}$ of 6-OHDA was dissolved into $200 \mu \mathrm{L}$ of saline containing $100 \mu \mathrm{g} / \mathrm{mL}$ L-ascorbate (Sigma Chemical Co.-Aldrich, St. Louis, MO; \#A-0278). This solution was then diluted into culture medium to a final concentration of between 0 and $500 \mu \mathrm{M}$. The resulting solution was added to the siRNA-treated cells on 96-well plates which were incubated at $37^{\circ} \mathrm{C}$ for $24 \mathrm{~h}$.

Alamar blue assay. The metabolic viability of neuroblastoma cells treated with siRNA and 6-OHDA was determined using the Alamar blue assay (Invitrogen Biosource, Carlsbad, CA; \#DAL1100). Alamar blue dye was diluted to $10 \% \mathrm{vol} / \mathrm{vol}$ in cell culture medium and cells were treated for $1 \mathrm{~h}$ at $37^{\circ} \mathrm{C}$, at which time the fluorescence in each well was determined using a Molecular Devices SpectraMax M5 plate reader at an excitation wavelength of $530 \mathrm{~nm}$ and an emission wavelength of $590 \mathrm{~nm}$. Six fluorescence measurements were taken per well and the mean and SD were calculated.

Protein isolation. Cells were washed with Hank's Balanced Salt Solution (Cellgro, Manassas, VA; \#21-022-CV) and then trypsinized (Cellgro, Manassas, VA; \#MT25-053-CI) for $5 \mathrm{~min}$ at $37^{\circ} \mathrm{C}$. The cells were then pipetted into a conical tube and centrifuged at $250 \times \mathrm{g}$ for $3 \mathrm{~min}$ and the supernatant was removed. The pellet was resuspended in $100 \mu \mathrm{L}$ RIPA buffer $[10 \mathrm{mM}$ Tris, pH 8 (MP Biomedicals, Solon, OH; \#819623); $150 \mathrm{mM} \mathrm{NaCl}$ (EMD Biosciences, Madison, WI; \#7760); 0.1\% vol/vol Nonidet P-40 (Sigma Chemical Co.-Aldrich, St. Louis, MO; \#I-8896); 0.5\% wt/vol sodium deoxycholate (Sigma Chemical Co.-Aldrich, St. Louis, MO; \#D-7650); and 0.1\% wt/vol SDS (Bio-Rad, Hercules, CA; \#161-0302)] supplemented with $4 \mu \mathrm{g} / \mathrm{mL}$ aprotinin (Sigma Chemical Co.-Aldrich, St. Louis, MO; \#A-6279), $1 \mathrm{mM}$ phenylmethanesulphonylfluoride (G-Biosciences, MD Heights, MO; \#786055), and $1 \mathrm{mM}$ sodium orthovanadate (Sigma Chemical Co.-Aldrich, St. Louis, MO; \#S-6508). The suspension was then sonicated through 10 cycles with a Branson Sonifier 450 on a constant output setting of 2 and incubated at $4^{\circ} \mathrm{C}$ for $30 \mathrm{~min}$ vortexing every $10 \mathrm{~min}$. The resulting mixture was centrifuged at $10,000 \times \mathrm{g}$ for $15 \mathrm{~min}$ and the supernatant was removed. The protein concentration was measured with a protein assay kit (Bio-Rad, Hercules, CA; \#500-0006).

Western blotting. Separating and stacking acrylamide gels were poured [7.5-12\% separating gel: $7.5-12 \%$ vol/vol acrylamide/bis (Bio-Rad, Hercules, CA; \#161-0154); 375 mM Tris, pH 8.8 (MP Biomedicals, Solon, OH; \#819623); 0.05\% wt/vol ammonium persulfate (VWR, Batavia, IL; \#VW1469-04); 0.05\% vol/vol TEMED (Bio-Rad, Hercules, CA; \#161-0800); $4 \%$ stacking gel: $4 \% \mathrm{vol} / \mathrm{vol}$ acrylamide/bis; $375 \mathrm{mM}$ Tris, $\mathrm{pH} 6.8 ; 0.05 \%$ $\mathrm{wt} / \mathrm{vol}$ ammonium persulfate; and $0.1 \%$ TEMED]. Protein samples were denatured and loaded onto the gels at 50-150 $\mu \mathrm{g}$ protein/lane and were run at $60 \mathrm{~V}$ for $30 \mathrm{~min}$ and then $90 \mathrm{~V}$ for $2 \mathrm{~h}$. Each gel was then transferred onto a nitrocellulose membrane at $90 \mathrm{~V}$ for $1.5 \mathrm{~h}$ on ice. The membrane was then blocked for $1 \mathrm{~h}$ at room temperature in blocking buffer [5\% dry milk (Bio-Rad, Hercules, CA; \#170-6404); $1 \times$ PBS (Sigma Chemical Co.-Aldrich, St. Louis, MO; D-5652)] on an orbital shaker. Primary antibodies, all purchased from Santa Cruz, except for anti-p75NTR (Promega, Madison, WI; \#G323A), were added at dilutions ranging from 1:200-1:1000 and the membranes were allowed to incubate at $4^{\circ} \mathrm{C}$ overnight on an orbital shaker. Membranes were washed twice with shaking for 10 min each with washing buffer $[0.1 \%$ vol/vol Tween-20 (Fisher Scientific, Hampton, NH; \#BP337$500)$ diluted in $1 \times$ PBS]. The secondary antibody was added in a ratio of 1:5000 in blocking buffer and the membrane and overlying solution were incubated at room temperature for $1 \mathrm{~h}$ on an orbital shaker. The membranes were washed with washing buffer four times for $10 \mathrm{~min}$ each at room temperature on an orbital shaker. The membranes were then placed on Saran Wrap and a chemiluminescent solution (Santa Cruz Biotechnology, Santa
Cruz, CA; \#sc-2048) was added. The excess solution was wiped away, and the membranes were exposed onto Kodak Biomax film (Kodak, Rochester, NY; \#165-1454). The films were then digitally scanned as TIFs.

Western blot band quantification and statistical analysis. Scion Imaging Software was used to quantify the TIF image bands. Background measurements were subtracted from each band $\mathrm{OD}$ and the results were normalized to an actin loading control band. A univariate ANOVA (two-way) with a post hoc Fisher's Least Significant Difference test was used to determine the statistical significance of the difference between pairs of samples at each concentration of 6-OHDA or pairs of Western blot bands. Statistical significance was assigned to differences with $p$ values of no greater than 0.01 .

\section{RESULTS}

The expression and relative stoichiometry of p75NTR and TrkA. The native expression of p75NTR and TrkA neurotrophin receptors and the resultant p75NTR:TrkA ratio vary widely across neural crest tumor cell lines (Fig. 1). Figure $1 A$ is a Western blot stained with antibodies for p75NTR and TrkA, respectively. Optical densitometry demonstrates the differential intensity of bands for each receptor in each cell line. However, as each antibody has a different affinity for its corresponding receptor and for secondary antibody, the relative OD of the p75NTR and TrkA bands in a given cell line may not accurately reflect the relative receptor number. We have previously demonstrated that the PC12 rat pheochromocytoma line used in these studies has a p75NTR:TrkA ratio of $\sim 100$ :1 (10). Using this and the OD data, we calculated the abundance of each receptor in each cell line. Figure $1 B$ shows the calculated p75NTR:TrkA ratios obtained. The neuroblastoma lines studied were divisible into two statistically distinct groups: those with high p75NTR:TrkA (Neuro-2A, SH-EP1) and those with low p75NTR:TrkA (SH-SY5Y, SK-N-AS) ratios.

Necdin mRNA is present in all neuroblastoma cell lines studied. The Neuro-2A, SH-EP1, SH-SY5Y, and SK-N-AS cell lines were shown by RT-PCR to express necdin (Fig. 2, $+\mathrm{RT})$. Primary cortical neurons were known to express necdin (11) and were used as a positive control. ATCC PC12 cells were known to lack necdin expression $(9,12)$ and were used as a negative control. The $-\mathrm{RT}$ lanes did not have reverse transcriptase during the production of cDNA and were used as controls to ensure that the samples did not contain genomic DNA.
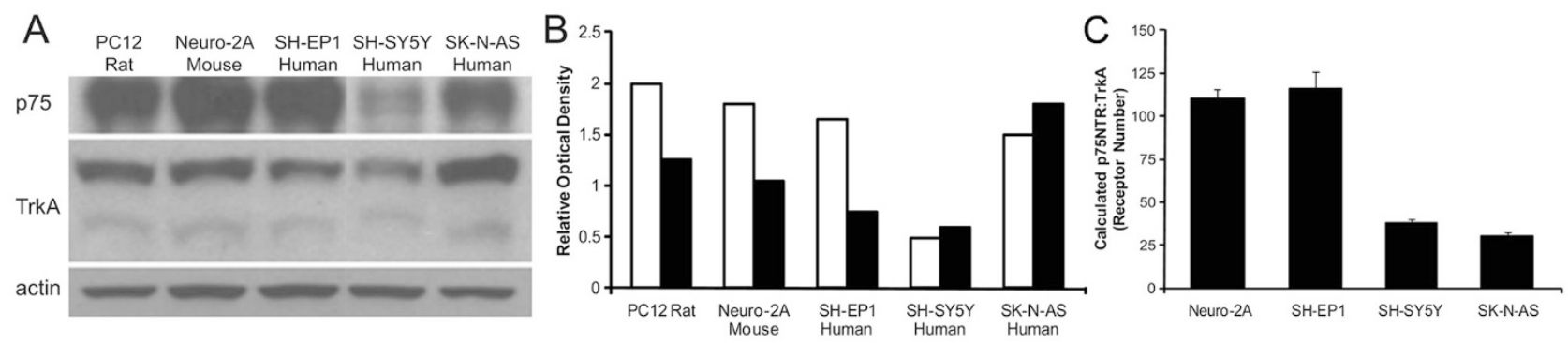

Figure 1. The p75NTR and TrkA expression and their relative stoichiometry in five neuroblastoma cell lines. (A) Western blots of lysates of four neuroblastoma cell lines and one pheochromocytoma cell line were analyzed by Scion Imaging (as described in Methods). (B) The OD values for the p75NTR and TrkA bands were normalized to their respective actin controls. $\square$, p75NTR; $\mathbf{\square}$, TrkA. $(C)$ The p75NTR:TrkA receptor number ratio was calculated from the OD values assuming a p75NTR:TrkA ratio of 100:1 for the PC12 pheochromocytoma cells. The ratios for Neuro-2A and SH-EP1 cells differ from those for SH-SY5Y and SK-N-AS cells with a $p$ value $<0.001$ (univariate two-way ANOVA, $n=$ Western blots on four independent samples). 

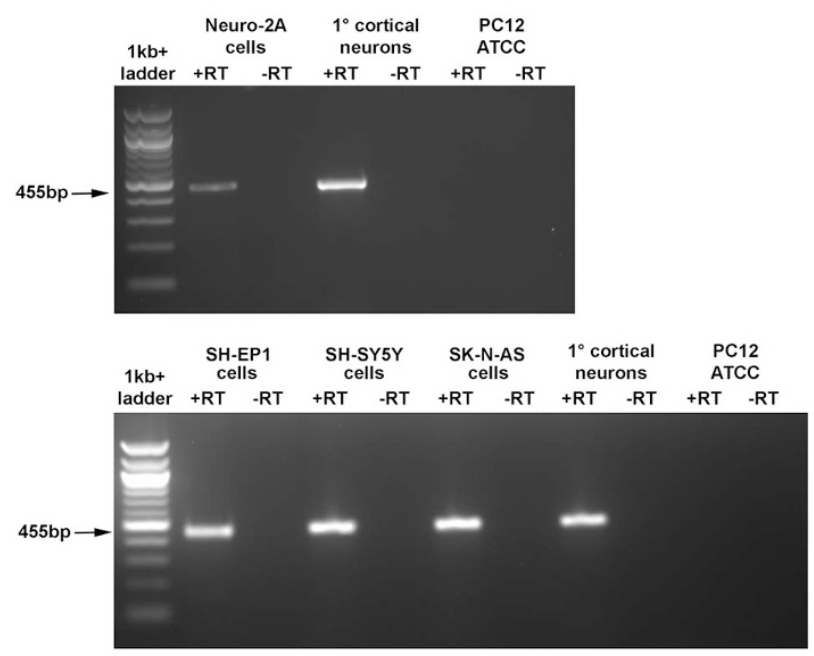

Figure 2. Necdin mRNA is present in all neuroblastoma cell lines used in this study. RT-PCR: A polymerase chain reaction amplifying a 455-bp fragment of necdin was carried out with cDNA from independent samples. A reverse transcriptase reaction was performed on mRNA isolated from Neuro2A, SH-EP1, SH-SY5Y, SK-N-AS, ATCC PC12, and primary cortical neuronal cells as described in the Materials and Methods. The primary cortical neurons were used as a positive control and the ATCC PC12 cells were used as a negative control for the presence of necdin mRNA. The absence of reverse transcriptase ( $-\mathrm{RT}$ ) was used as a control to show there was no genomic DNA contamination in the mRNA samples before making the cDNA.

A

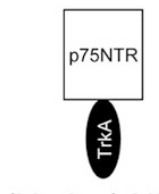
(Native) High p75NTR:TrkA Neuro-2A; SH-EP1

B

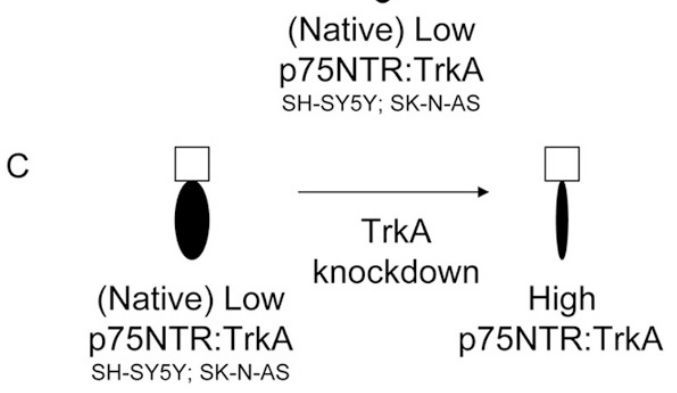

Figure 3. Native and engineered cell lines used to test the hypothesis that the p75NTR:TrkA ratio determines the relationship between necdin expression and sensitivity to oxidant stress. Testing this hypothesis required differentiating the effects of altered p75NTR:TrkA ratio from those of differing p75NTR or TrkA content per se. We therefore used the following native or engineered neuroblastoma cell lines: High p75NTR:TrkA lines (Neuro-2A; SH-EP1; SH-SY5Y TrkA knockdown; SK-N-AS TrkA knockdown) and low p75NTR:TrkA lines (SH-SY5Y; SK-N-AS; Neuro-2A p75NTR knockdown; SH-EP1 p75NTR knockdown). In each case, the cartoon depicts the ratio of p75NTR:TrkA using white squares for p75NTR and black ellipses for TrkA. Changes in the size of the squares and ellipses symbolize molecularly engineered changes in expression of p75NTR and TrkA, respectively.
p75NTR:TrkA ratio-dependence of effects of necdin knockdown on sensitivity to oxidant stress. To determine the relationship between necdin content and sensitivity to oxidant stress in cells of differing p75NTR:TrkA ratios, we used two different groups of cell lines (Fig. 3): high p75NTR:TrkA lines (Neuro-2A; SH-EP1; SH-SY5Y TrkA knockdown; SK-N-AS TrkA knockdown) and low p75NTR:TrkA lines (SH-SY5Y; SK-N-AS; Neuro-2A p75NTR knockdown; SH-EP1 p75NTR knockdown). These native and engineered cell lines allowed us to distinguish the effects of changing p75NTR or TrkA content per se from the effects of changing the ratio of p75NTR to TrkA. The studies described below demonstrate that it is the ratio of p75NTR to TrkA that determines the effects of varying necdin content on sensitivity to oxidant stress.

In Neuro-2A and SH-EP1 cells, lines with high p75NTR:TrkA ratios, knockdown of necdin was without effect on the concentration-response curve to 6-OHDA. Neuro-2A p75NTR knockdown and SH-EP1 p75NTR knockdown cells had both decreased p75NTR content and decreased p75NTR:TrkA ratio. These cells demonstrated increased sensitivity to 6-OHDA relative to their native counterparts (Fig. $4 A$ and $B$ ). Subsequent knockdown of necdin in these p75NTR-deficient cells decreased the sensitivity to oxidant stress in the direction of that of the native cells. Although this demonstrates change in necdin-dependence with decreased p75NTR, from this experiment, we could not determine whether lowering of p75NTR content per se or lowering of the p75NTR:TrkA ratio was responsible for the change in the effects of necdin knockdown on sensitivity to oxidant stress.

SH-SY5Y and SK-N-AS human neuroblastoma cells both have low p75NTR:TrkA ratios (Figs. 1 and 3). However, SH-SY5Y cells have low p75NTR content; SK-N-AS cells have high p75NTR content (Fig. 1). Comparing these two cell lines might help differentiate the impact of p75NTR content from that of p75NTR:TrkA ratio on necdin-dependence of sensitivity to oxidant stress.

Lowering p75NTR further with siRNA did not affect sensitivity to 6-OHDA in either cell line (Fig. $4 C$ and $D$ ). Similar to the case for Neuro-2A and SH-EP1 cells after p75NTR knockdown, in both SH-SY5Y and SK-N-AS cells, necdin knockdown decreased sensitivity to oxidant stress. This suggests that it is the p75NTR:TrkA ratio and not the p75NTR content per se determines the effects of necdin knockdown on sensitivity to oxidant stress.

We tested this hypothesis by knocking down TrkA in low p75NTR:TrkA cells (Fig. 3). We predicted that this would decrease sensitivity of the cells to oxidant stress. We further predicted that, as is the case for native high p75NTR:TrkA cells, knockdown of necdin would be without effect on sensitivity to 6-OHDA; knockdown of p75NTR would enhance sensitivity to 6-OHDA; and knockdown of necdin after knockdown of p75NTR would return sensitivity to 6-OHDA to control levels.

A partial knockdown of TrkA expression increased the ratio of p75NTR:TrkA (Figs. 3 and 5A). This effectively reduces the TrkA expression 2- to 3-fold, thereby increasing 2- to 3-fold the p75NTR:TrkA ratio of SH-SY5Y and SK-N-AS 

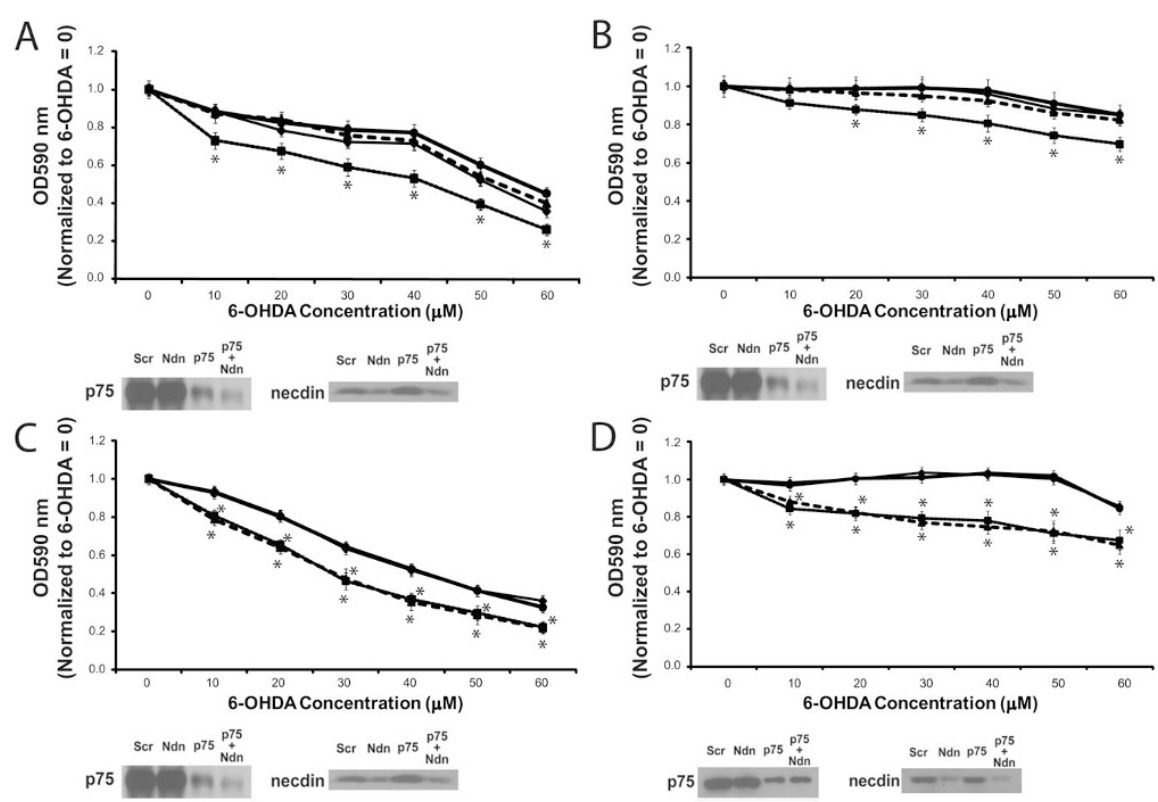

Figure 4. Concentration-response curves to assess the effects of 6-hydroxydopamine (6-OHDA) on the viability of neuroblastoma cells with altered content of p75NTR and/or necdin. Each of four neuroblastoma cell lines were transfected with scrambled, necdin, p75NTR, or (p75NTR + necdin) siRNA, respectively, and treated with 6-OHDA. Alamar blue staining was performed as described in Methods and $\mathrm{OD}_{590 \mathrm{~nm}}$ values obtained at each concentration of 6-OHDA were normalized to the value obtained with $0 \mu \mathrm{M}$ 6-OHDA treatment for all conditions. $\boldsymbol{\Lambda}$, scrambled siRNA; $\bullet$, necdin siRNA; $\mathbf{\square}$, p75NTR siRNA;, p75NTR siRNA + necdin siRNA. ${ }^{*} p<0.01$ (univariate two-way ANOVA, $n=16$, four independent runs with four repeats each). $A$, Neuro-2A cells; $B$, SH-EP1 cells; $C$, SH-SY5Y cells; $D$, SK-N-AS cells. $A, B$ : high p75NTR:TrkA cell lines; $C, D$ : low p75NTR:TrkA cell lines.
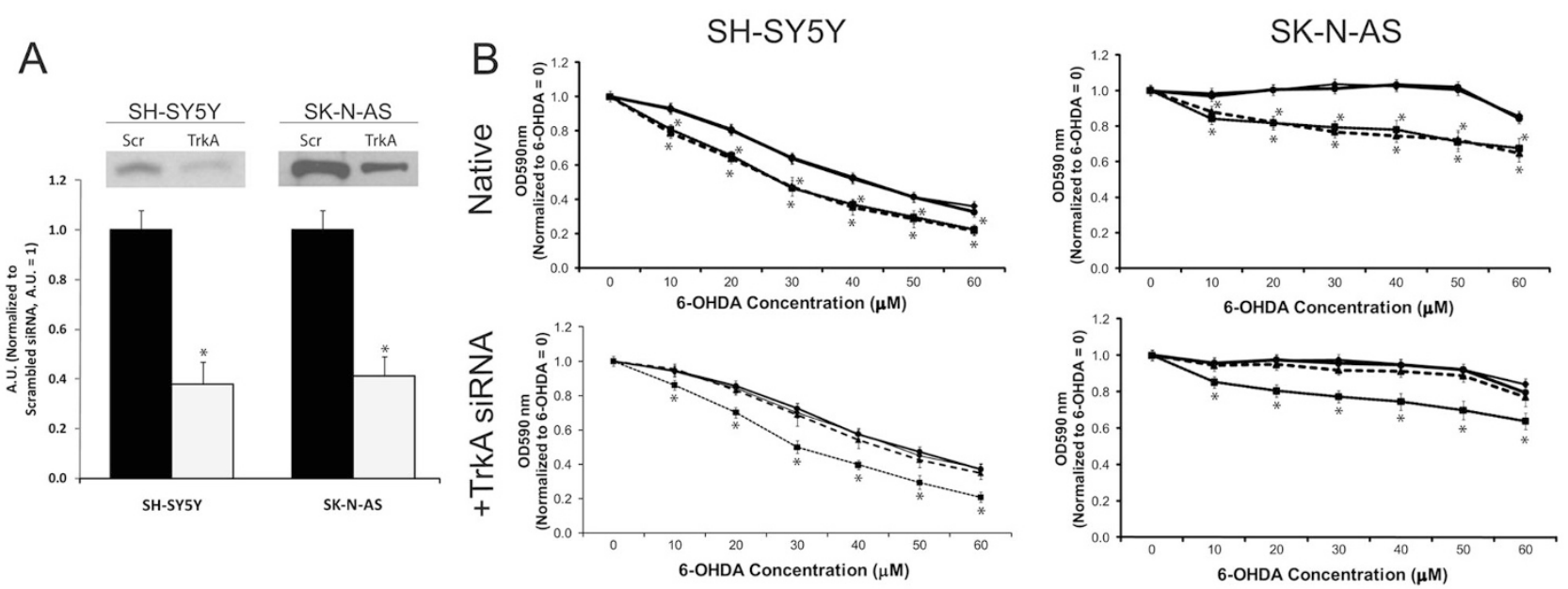

Figure 5. Effects of TrkA knockdown on the 6-OHDA concentration-response curves of low p75NTR:TrkA ratio cells. (A) SH-SY5Y and SK-N-AS cells were treated with either scrambled siRNA (black bars) or TrkA siRNA (white bars) and subjected to Western blotting for TrkA. Western blots were analyzed by Scion Imaging (as described in Methods). The OD values for the TrkA bands were normalized to their respective actin controls (actin blot not shown). The scrambled siRNA-treated control cell values were set to 1 and the TrkA siRNA-treated cell values were expressed as a function of the control. * $p<0.01$ (univariate two-way ANOVA, $n=$ Western blots of three independent samples). (B) SH-SY5Y and SK-N-AS cells, in their native state or stably transfected with TrkA siRNA, were transfected with scrambled $(\boldsymbol{\Delta})$, necdin $(\bullet)$, p75NTR $(\mathbf{\square})$, or [p75NTR + necdin] $(\bullet)$ siRNA, respectively, and treated with 6-OHDA. Alamar blue staining was performed as described in Methods and $\mathrm{OD}_{590 \mathrm{~nm}}$ values obtained at each concentration of 6-OHDA were normalized to the value obtained with $0 \mu \mathrm{M}$ 6-OHDA treatment for all conditions. ${ }^{*} p<0.01$ (univariate two-way ANOVA, $n=16$, four independent runs with four repeats each). Note that the plots for native cells were also shown in Figure $4 C$ and $D$ and are repeated here for ease of comparison.

neuroblastoma cells, giving them the same ratio observed in SH-EP1 cells (Figs. 1 and 3). As predicted, after TrkA knockdown, SH-SY5Y and SK-N-AS cells respond to p75NTR and/or necdin knockdown in a way that resembles Neuro-2A and SH-EP1 cells (Fig. 5B). Changing the ratio of p75NTR:TrkA without changing the p75NTR content was sufficient to change the effects of necdin down-regulation on sensitivity to oxidant stress.

Effects of necdin knockdown on TrkA expression and signaling. In contrast to the trophic role of TrkA signaling in normal neurons, TrkA signaling is proapoptotic in many tumor cell lines $(6,13-15)$. We therefore hypothesized that, in low p75NTR:TrkA ratio cells, necdin knockdown correlates with down-regulation of TrkA and decreased TrkA signaling.

Figure 6 demonstrates that, in native SH-SY5Y and SK-N-AS cells and in Neuro-2A p75NTR knockdown and SH-EP1 p75NTR knockdown cells (Fig. 3), down-regulation of necdin results in down-regulation of TrkA and a decrease in the ratios of phosphorylated to nonphosphorylated TrkA (pTrkA/TrkA), phosphorylated to nonphosphorylated c-Jun-N-terminal kinases (pJNK/JNK), and phosphorylated to nonphosphorylated extracellular signal-regulated kinases (pERK/ERK), all markers of TrkA signaling. In addition, in concert with their respective responses to oxidant stress, scrambled siRNA-treated 

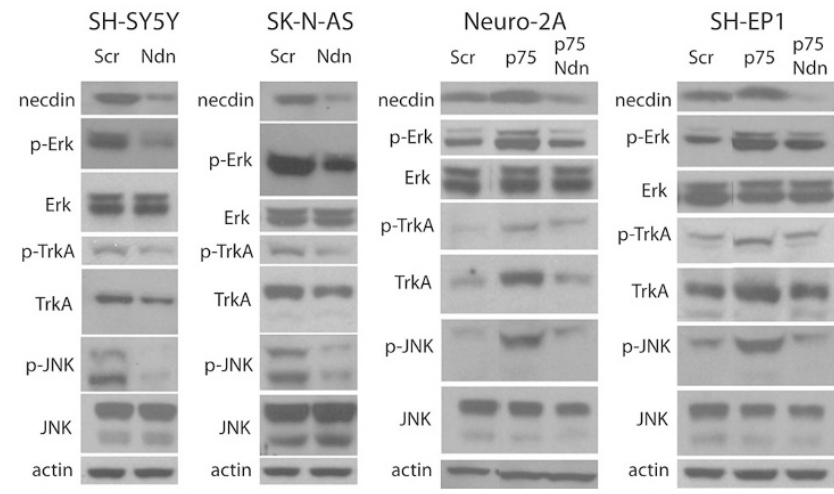

Figure 6. Western blots for TrkA signal transductants in low p75NTR:TrkA ratio cells. Low p75NTR:TrkA cells, whether in their native state or engineered by knockdown of p75NTR, were assayed by Western blotting for TrkA signal transductants after transfection with either a control construct or necdin (Ndn) siRNA. In the case of native SH-SY5Y and SK-N-AS cells, the control construct was scrambled (Scr) siRNA. In the case of Neuro-2A and SH-EP1 cells in which p75NTR had to be knocked down to make them low p75NTR:TrkA cells, the control construct was p75NTR siRNA alone. Results for scrambled siRNA-treated Neuro-2A and SH-EP1 cells are also shown, demonstrating that necdin depletion of engineered p75NTR-deficient cells of these lines restores their TrkA signaling status to that of the scrambled siRNA-treated cells.

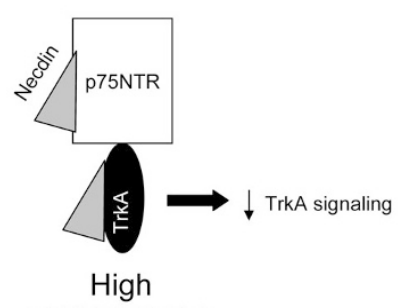

p75NTR:TrkA

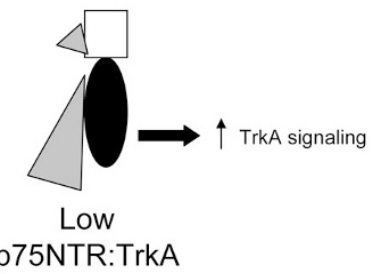

Figure 7. Necdin apportionment between p75NTR and TrkA. The mechanism that ties together the p75NTR:TrkA ratio dependence of the effects of necdin on sensitivity to oxidative stress and the necdin dependence of TrkA signaling magnitude seen in normal neurons is not known. One possibility is that decreasing p75NTR (white squares) expression decreases the proportion of total necdin (gray triangles) that can bind to p75NTR. The "liberated" necdin is then available to bind to TrkA (black ellipses), shifting the necdin binding ratio in favor of TrkA and enhancing TrkA signaling.

Neuro-2A and SK-N-AS cells do not signal through TrkA as robustly as Neuro-2A p75NTR knockdown and SH-EP1 p75NTR knockdown cells.

\section{DISCUSSION}

The expression of p75NTR enhances the resistance of neuroblastoma cells to 6-OHDA by a mechanism that includes decreased TrkA expression (13), enhanced glutathione recycling (10), and activation of the PI3 K/AKT pathway (16). The mechanism by which p75NTR and TrkA are coordinately regulated is not well understood. Although TrkA is known to prevent apoptosis and increase cell survival in a number of cell types (17-19), TrkA can also lead to cell death in neuroblastomas and medulloblastomas $(6,13,14,20)$. A high p75NTR:TrkA ratio enhances resistance to 6-OHDA and reduces TrkA-induced cell death. A low p75NTR:TrkA ratio increases TrkA activation, leading to both ERK and JNK phosphorylation and effecting subsequent cell death (13). Increasing the p75NTR:TrkA ratio of low p75NTR:TrkA cells enhances their resistance to oxidant stress.

Increased expression of the MAGE interactor protein, necdin increases TrkA signaling in normal neurons. Necdin is known to bind to p75NTR, TrkA, and their heterocomplex (9). Down-regulation of p75NTR might shift binding of necdin to TrkA, facilitating robust NGF-mediated TrkA activation and signaling (Fig. 7). We previously demonstrated that necdin knockdown prevents TrkA activation and cell death in p75NTR-deficient PC12 rat pheochromocytoma cells (13).

The data presented here demonstrate the relevance of these findings for human neural crest cells and the role of the p75NTR:TrkA ratio in determination of the effects of necdin deficiency on resistance to oxidant stress. Alteration of the p75NTR:TrkA ratio, whether by manipulation of the cellular content of p75NTR or TrkA, alters sensitivity to oxidant stress. In cells with a low p75NTR:TrkA ratio and consequent high sensitivity to oxidant stress, necdin knockdown results in decreased TrkA signaling and consequent decrease in sensitivity to oxidant stress.

The differential effects of necdin deficiency in cells with high or low p75NTR:TrkA ratios, respectively, is of potential interest for the developmental disorder, Prader-Willi syndrome. Patients with Prader-Willi syndrome have deletions of the paternal gene for necdin and are necdin-deficient (21). The necdin gene is heavily imprinted and the paternal allele determines necdin expression level. The effect of necdin deficiency on neurons of a particular brain or PNS region may well depend on the p75NTR:TrkA ratio of these neurons.

Neurotrophin receptors have also been implicated in susceptibility of neuroblastoma cells to chemotherapeutic attack $(2,4-$ $7,22,23)$. Previous studies have demonstrated the relevance of p75NTR:TrkA ratio in determination of neuroblastoma chemosensitivity. This work suggests that p75NTR:TrkA ratio alone is insufficient as a biomarker of chemosensitivity, as so-called interactor proteins, like necdin, can modify chemosensitivity at a given p75NTR:TrkA ratio. The definitive demonstration of the differential apportionment of necdin between p75NTR and TrkA in cells with different p75NTR:TrkA ratios, definition of the importance of necdin as an interactor with p75NTR and TrkA for normal central and peripheral neurons, and determination of the mechanism by which necdin binding enhances TrkA signaling will be the aims of future studies.

Acknowledgments. We thank Candy Hom and Dr. Robert $\mathrm{H}$. Schor for their assistance in graphic illustration.

\section{REFERENCES}

1. Rogers D, Schor NF 2010 The child is father to the man: developmental roles for proteins of importance for neurodegenerative disease. Ann Neurol 67:151-158

2. Schor NF 2005 p75NTR in development and disease. Prog Neurobiol 77:201-214

3. Schor NF 2001 Signal transduction for clinicians: why should we care? Pediatr Neurol 25:361-367

4. Cortazzo MH, Kassis ES, Sproul KA, Schor NF 1996 Nerve growth factor (NGF)mediated protection of neural crest cells from antimitotic agent-induced apoptosis. J Neurosci 16:3895-3899

5. Yan C, Liang Y, Nylander KD, Wong J, Rudavsky RM, Saragovi HU, Schor NF 2002 p75-NGF as an antiapoptotic complex: independence vs. cooperativity. Mol Pharmacol 61:710-719 
6. Yan C, Liang Y, Nylander KD, Schor NF 2002 TrkA as a life and death receptor: receptor dose as a mediator of function. Cancer Res 62:4867-4875

7. Yan C, Mirnics ZK, Portugal CF, Liang Y, Nylander KD, Rudzinski M, Zaccaro C Saragovi HU, Schor NF 2005 Cholesterol biosynthesis and the pro-apoptotic effect of the p75 nerve growth factor receptor. Brain Res Mol Brain Res 139:225-234

8. Korade Z, Mi Z, Schor NF 2007 Expression and p75 neurotrophin receptor dependence of cholesterol synthetic enzymes in adult mouse brain. Neurobiol Aging 28:1522-1531

9. Kuwako K, Hosokawa A, Nishimura I, Uetsuki T, Yamada M, Nada S, Okada M, Yoshikawa K 2005 Disruption of the paternal necdin gene diminishes TrkA signaling for sensory neuron survival. J Neurosci 25:7090-7099

10. Tyurina YY, Nylander KD, Mirnics ZK, Portugal C, Yan C, Zaccaro C, Saragovi HU, Kagan VE, Schor NF 2005 The intracellular domain of p75NTR as a determinant of cellular reducing potential and response to oxidant stress. Aging Cell 4:187-196

11. Aizawa T, Maruyama K, Kondo H, Yoshikawa K 1992 Expression of necdin, an embryonal carcinoma-derived nuclear protein, in developing mouse brain. Brain Res Dev Brain Res 68:265-274

12. Tcherpakov M, Bronfman FC, Conticello SG, Vaskovsky A, Levy Z, Niinobe M, Yoshikawa K, Arenas E, Fainzilber M 2002 The p75 neurotrophin receptor interacts with multiple MAGE proteins. J Biol Chem 277:49101-49104

13. Ingraham CA, Schor NF 2009 Necdin and TrkA contribute to modulation by p75NTR of resistance to oxidant stress. Exp Cell Res 315:3532-3542

14. Muragaki Y, Chou TT, Kaplan DR, Trojanowski JQ, Lee VM 1997 Nerve growth factor induces apoptosis in human medulloblastoma cell lines that express TrkA receptors. J Neurosci 17:530-542
15. Harel L, Costa B, Fainzilber M 2010 On the death Trk. Dev Neurobiol 70:298-303

16. Mi Z, Rogers DA, Mirnics ZK, Schor NF 2009 p75NTR-dependent modulation of cellular handling of reactive oxygen species. J Neurochem 110:295-306

17. Eggert A, Sieverts H, Ikegaki N, Brodeur GM 2000 p75 mediated apoptosis in neuroblastoma cells is inhibited by expression of TrkA. Med Pediatr Oncol 35:573576

18. Arévalo JC, Waite J, Rajagopal R, Beyna M, Chen ZY, Lee FS, Chao MV 2006 Cell survival through Trk neurotrophin receptors is differentially regulated by ubiquitination. Neuron 50:549-559

19. Com E, Lagadec C, Page A, El Yazidi-Belkoura I, Slomianny C, Spencer A, Hammache D, Rudkin BB, Hondermarck H 2007 Nerve growth factor receptor TrkA signaling in breast cancer cells involves Ku70 to prevent apoptosis. Mol Cell Proteomics 6:1842-1854

20. Lavoie JF, Lesauteur L, Kohn J, Wong J, Furtoss O, Thiele CJ, Miller FD, Kaplan DR 2005 TrkA induces apoptosis of neuroblastoma cells and does so via a p53-dependent mechanism. J Biol Chem 280:29199-29207

21. MacDonald HR, Wevrick R 1997 The necdin gene is deleted in Prader-Willi syndrome and is imprinted in human and mouse. Hum Mol Genet 6:1873-1878

22. Zhang T, Mi Z, Schor NF 2009 Role of tyrosine phosphorylation in the antioxidant effects of the p75 neurotrophin receptor. Oxid Med Cell Longey 2:238-246

23. Bassili M, Birman E, Schor NF, Saragovi HU 2010 Differential roles of Trk and p75 neurotrophin receptors in tumorigenesis and chemoresistance ex vivo and in vivo. Cancer Chemother Pharmacol 65:1047-1056 